\title{
Resting functional connectivity and mild cognitive impairment in Parkinson's disease. An electroencephalogram study
}

\author{
Jairo Alexander Carmona Arroyave*,1,2, Carlos Andrés Tobón Quintero ${ }^{1,2}$, Jasmín Jimena \\ Suárez Revelo ${ }^{1,3}$, John Fredy Ochoa Gómez ${ }^{1,3}$, Yamile Bocanegra García1,2, Leonardo \\ Moreno Gómez ${ }^{5}$ \& David Antonio Pineda Salazar ${ }^{1,2,4}$ \\ ${ }^{1}$ Neuroscience Group, Medical School, University of Antioquia, SIU, Calle 62 №52-59, Medellín, Colombia \\ ${ }^{2}$ Neuropsychology \& Behavior Group (GRUNECO), Medical School, University of Antioquia, SIU - Área Asistencial, Calle 62 No. \\ 52-59, Medellín, Colombia \\ ${ }^{3}$ Bioinstrumentation \& Clinical Engineering Research Group (GIBIC), Bioengineering Program, University of Antioquia, Calle 70 No. \\ 52-21, Medellín, Colombia \\ ${ }^{4}$ Neuropsychology \& Behavior Group (GRUNECO), Psychology Department, University of San Buenaventura, Carrera 56 C No. \\ 5-110, Medellín, Colombia \\ ${ }^{5}$ Neurology Unit. Pablo Tobón Uribe Hospital, Calle 78B No. 69-240, Medellín, Colombia \\ *Author for correspondence: Tel.: (+574) 21964 44; Fax: (+574) 21964 44; jairo.carmona@udea.edu.co
}

Objective: Parkinson's disease (PD) is characterized by cognitive deficits. There is not clarity about electroencephalogram (EEG) connectivity related to the cognitive profile of patients. Our objective was to evaluate connectivity over resting EEG in nondemented PD. Methods: PD subjects with and without mild cognitive impairment $(\mathrm{MCl})$ were assessed using coherence from resting EEG for local, intra and interhemispheric connectivity. Results: PD subjects without $\mathrm{MCI}$ (PD-nMCI) had lower intra and interhemispheric coherence in alpha2 compared with controls. PD with $\mathrm{MCI}$ (PD-MCl) showed higher intra and posterior interhemispheric coherence in alpha2 and beta1, respectively, in comparison to PD-nMCl. PD-MCI presented lower frontal coherence in beta frequencies compared with PD-nMCl. Conclusion: EEG coherence measures indicate distinct cortical activity in PD with and without $\mathrm{MCl}$.

First draft submitted: 23 December 2018; Accepted for publication: 13 March 2019; Published online: 24 May 2019

Keywords: cognitive impairment $\bullet$ electroencephalography $\bullet$ executive functions $\bullet$ functional connectivity $\bullet$ Parkinson's disease $\bullet$ resting state

Parkinson's disease (PD) is the second most frequent neurodegenerative disorder in elder population [1,2]. Disease progression impacts quality of life of patients and caregivers owing to a combination of motor and nonmotor dysfunctions [3-6]. Cognitive disturbances are frequent in PD since the diagnosis [7-10], ranging from subjective complains and mild cognitive impairment (MCI) to the presentation of a definite dementia syndrome [11,12]. MCI is recognized as a risk factor for developing dementia in PD [13,14], making urgent the identification of reliable markers to assist clinicians in diagnosing or monitoring patients at increased hazard of worse prognosis [15].

Cognitive impairment in PD involves alterations in several domains [16-18], starting more often with abnormalities in executive functions $[13,16,19,20]$. Disturbances in information storage, visoperceptual capacities and language, appear in more advanced stages, being indicative of an evolution to dementia [14,16,21]. These observations have led to the proposal of a dual syndrome hypothesis [22], which differentiates between an early frontal, predominantly dysexecutive and dopamine dependent syndrome, and a later more posterior syndrome, with prominent visuospatial and memory storage deficits, associated to cholinergic anomalies $[17,22,23]$. Pathophysiology of cognitive impairments in PD appear to entail disruptions in the cortico-subcortical circuits, which would explain the diversity of clinical features of disease [24-26].

The basis of the emergency of high-order cognitive processes is the integration of specialized brain areas through complex connectivity arrays conforming organized networks [27-30]. Neurophysiological correlates of those processes can be tracked through non-invasive techniques such as the electroencephalogram (EEG), evaluating synchronized

Future Medicine 
activity between neuronal populations [31-33]. The analytical approach that examines the statistical dependencies among signals from different neural units has been termed functional connectivity (FC) [32,34]. In the frame of global brain networks, abnormal activity of one circuit component could alter its interactions with other network components, which should be expressed as modulations in the brain-based FC measurements $[35,36]$.

Extracranial electrophysiological (EEG/MEG) studies have found connectivity changes in several nervous system disorders [37-40], including PD [41,42]. Resting-state reports in PD without dementia (PD-ND) have demonstrated that FC measures could distinguish patients from healthy controls [43-45], being also sensitive to different types of therapeutic interventions [46-48]. Connectivity and other EEG measures have been examined by means of the quantitative analysis of the EEG (qEEG) [49,50]. This computerized and mathematical spectral analysis of EEG allows to gain quantitative data and has shown diagnostic differentiation capacity in several neuropsychiatric conditions [50-54]. Nonetheless, evidence about EEG connectivity through the full cognitive spectrum in PD-ND is scarce, and the most used FC measure has been the spectral coherence [55-57]. In summary, there is a particular lack of data regarding correlations between EEG FC features and cognitive variables in PD populations [56,58,59].

The present study examine resting-state FC characteristics in PD subjects with and without MCI applying qEEG methods. We sought to determine corticocortical coherence patterns associated to cognitive status of patients with $\mathrm{PD}$, and to explore correlations between frontal measures and executive functioning.

\section{Materials \& methods \\ Subjects}

An initial group of 50 subjects with diagnosis of PD was recruited from the outpatient service of the neurosciences group of our university. All patients underwent a complete neurological and neuropsychological examination, in order to confirm diagnosis and to apply selection criteria. We excluded subjects with other parkinsonian syndromes, additional major neurological/psychiatric disorders, any kind of dementia [60], presence of intracranial devices and the current use of drugs that could alter the EEG. PD diagnosis was confirmed with the criteria of the United Kingdom PD Society Brain Bank [61], disease progression was established with the Hoehn \& Yahr scale (H\&Y) [62,63], and motor disability was determined with section III of the Unified Parkinson's Disease Rating Scale (UPDRS-III) [64]. After applying the selection criteria, $36(\mathrm{~N}=36)$ patients were selected to enter the study.

Cognitive screening was performed using the Montreal Cognitive Assessment (MoCA) [65], a reliable tool for the estimation of global cognitive state and the identification of MCI in PD [66,67], which has been validated in a Colombian population [68]. Subject's functionality were evaluated with the Barthel Index and Lawton \& Brody scale [69,70]. All patients were under stable antiparkinsonian treatment during at least 4 weeks prior to study evaluations, and individual medications was converted to levodopa-equivalent daily dose (LEDD) [71]. All clinical and electrophysiological assessments were completed in phase 'On' of levodopa treatment.

PD subjects were classified in two groups depending on their cognitive status. Patients with spared functionality, not significant cognitive complains, and a MoCA score of 23 or above (according to validation in Colombian population) [68], were classified as PD without MCI (PD-nMCI, $n=22)$. Patients with preserved functionality and a MoCA score below 23 were grouped as PD with MCI (PD-MCI, $n=14$ ), following level one of the diagnostic criteria for MCI in PD proposed by the Movement Disorders Society (MDS) [72].

Finally, $36(\mathrm{~N}=36)$ healthy subjects with normal cognitive status, and not relevant neurologic or psychiatric disorders (controls), were matched to PD patients based on gender, age and formal education. All controls were assessed cognitively in the same way described for patients.

The study protocol had the approval of the Ethical Research Committee of the University (Certificate No. 15-10-569). All participants gave their written informed consent after an explanation of the study characteristics, and before any clinical or experimental evaluation, according to the declaration of Helsinki.

\section{Executive function assessment}

All subjects were evaluated with the INECO frontal screening test (IFS) [73], a sensitive tool for detection of executive dysfunction in neurodegenerative diseases [74]. The IFS focus on core components of executive functions, as the adequacy of attentional resources, a proper operative memory, response inhibition capacity and abilities for behavior monitoring [75]. In correspondence, the IFS represents primarily three groups of tasks: set shifting and response inhibition, working memory and abstraction capacity. IFS total score (30 points) was obtained by the sum of its eight subtest punctuations [73]: Motor Programming (3 points), Conflicting Instructions ( 3 points), Go-No 
Go (3 points), Backward Digit Span (6 points), Verbal Working Memory (2 points), Visual Working Memory (4 points), Abstraction Capacity - Proverb Interpretation (3 points) and Verbal Inhibitory Control (6 points).

\section{EEG recordings}

EEG was recorded in resting awake condition with eyes closed during five minutes in a quiet and dimly shielded room. Subjects seated upright in a comfortable chair being instructed to be relaxed and to remain alert during recording. EEG Signals were acquired with a 58 tin electrodes cap according to the international 10-10 system, using Neuroscan@ software and amplifiers (Scan 4.5, SynAmps2). A sampling frequency of $1000 \mathrm{~Hz}$ was used to digitize signals, which were filtered online with a band-pass filter of 0.05 to $200 \mathrm{~Hz}$, and a notch filter of $60 \mathrm{~Hz}$. The reference acquisition consisted of an electrode placed on the right earlobe. As ground reference we used another electrode located between $\mathrm{Cz}$ and $\mathrm{Fz}$ positions. Impedances were kept below $10 \mathrm{kOhm}$.

\section{EEG data preprocessing}

A semi-automated pre-processing pipeline was implemented using two MATLAB toolboxes [76]: the EEGLAB [77], and the standardized early-stage EEG processing pipeline (PREP) [78]. This process comprises: data importation; visual inspection to remove segments representing abrupt movements; PREP pipeline, which includes a robust reference to average with exclusion of defective channels, and detection with interpolation of bad channels; filtering of data using a high pass FIR filter at $1 \mathrm{~Hz}$; independent component analysis (ICA); epoch segmentation to $2 \mathrm{~s}$; filtration of artifactual independent components using wavelet (wICA) to correct muscular and eye artifacts [79]; low-pass FIR filtering at $30 \mathrm{~Hz}$; and rejection of remaining deficient epochs by a procedure based on linear trend, joint probability and kurtosis approach [80]. On average, for each subject, $4.96( \pm 3.66)$ channels were interpolated, $9.0( \pm 3.09)$ epochs were rejected and $164.38( \pm 20.01)$ epochs were kept for subsequent analysis.

\section{FC analysis}

FC was assessed using the coherence function estimated by the multi-taper method [81], available in Chronux MATLAB toolbox [82]. Coherence measures the strength of the correlation between two signals across frequencies, quantifying the amount of dispersion of phases and amplitudes of the signals in a specific frequency width [83]. Coherence captures only the linear component of the functional coupling, and can be affected by volume conduction of remote sources [33]. Analysis of spectral coherence has been the predominant approach in qEEG reports in several clinical conditions, being the most common in studies of PD [84]. Thus, we used coherence analysis in an explorative manner because the introduction a cognitive subgroup of patients that have not been considered in previous EEG FC studies $[57,58,84]$.

Coherence was calculated between all pairs of electrodes within or between specified regions in four frequency bands: alpha1 $(8-10 \mathrm{~Hz})$, alpha2 $(10-13 \mathrm{~Hz})$, beta1 $(13-20 \mathrm{~Hz})$ and beta2 $(20-30 \mathrm{~Hz})$. Computations were made from 50 free-of-artifacts epochs randomly selected from each recording. The magnitude of coherence was averaged across epochs, resulting in a $58 \times 58$ matrix for each subject. To lessen comparisons, measurements were reduced to eight Regions of Interest (ROIs) based on the position of electrodes representing the area over the main brain lobes. Electrode sites were included in ROIs as follows: left frontal (AF3, F1, F3, FC1, FC3); right frontal (AF4, F2, F4, FC2, FC4); left temporal (FC5, C5, CP5, T7, TP7); right temporal (FC6, C6, CP6, T8, TP8); left parietal (CP1, CP3, P1, P3); right parietal (CP2, CP4, P2, P4); left occipital (PO3, PO5, PO7, O1); and right occipital (PO4, PO6, PO8, O2) (Figure 1A).

For each frequency band, three types of connectivity were estimated based on ROIs interplay. Local (IntraROI) connectivity was calculated averaging coherence values obtained from all pairwise channel combinations within each ROI. Intrahemispheric connectivity was determined by the average of coherence among all electrode pairs between two ROIs in the same hemisphere. Interhemispheric connectivity was established by averaging coherence from every pair of channels between homolog ROIs of both hemispheres. ROIs and selected interactions according to connectivity types are shown in Figure 1.

\section{Statistical analysis}

\section{Demographic \& clinical characterization}

Demographic and clinical characteristics were compared between groups. Normal distribution of continuous variables was evaluated by Shapiro-Wilk normality test. If data sample was normally distributed, parametric 
(A)

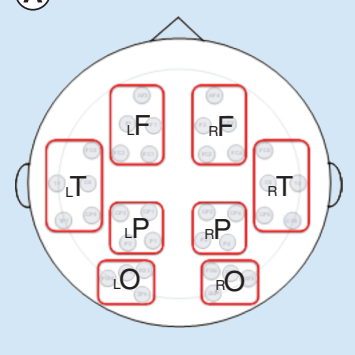

(B)

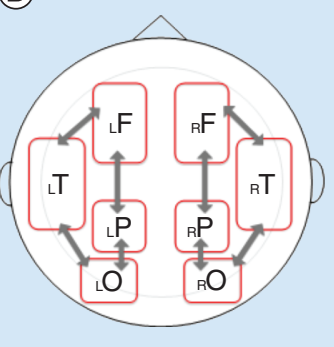

(c)

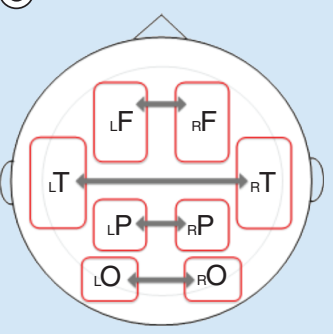

Figure 1. Schematic representation of the electroencephalogram regions of interest.

Table 1. Demographic, clinical and cognitive characterization of Parkinson's disease groups and healthy controls.

\begin{tabular}{|c|c|c|c|c|c|c|}
\hline $\begin{array}{l}\text { Demographic and } \\
\text { clinical variables }\end{array}$ & Controls $(n=36)$ & PD-nMCI $(n=22)$ & PD-MCI $(n=14)$ & $\mathbf{F}^{\dagger}$ & $p$ value & Group comparisons \\
\hline Age (years) & $63.3(6.2)$ & $61.8(8.0)$ & $66.1(7.4)$ & 1,559 & ANOVA 0.218 & \\
\hline Gender $(F \backslash M)$ & $12 \backslash 24$ & $8 \backslash 14$ & $4 \backslash 10$ & & Fisher's exact 0.946 & \\
\hline Education (years) & $12.4(4.8)$ & $11.5(5.0)$ & $11.9(5.6)$ & 0.224 & ANOVA 0.800 & \\
\hline $\begin{array}{l}\text { Disease duration } \\
\text { (years) }\end{array}$ & & $4.4(2.7)$ & $6.2(3.5)$ & 2898 & ANOVA 0.098 & \\
\hline Hoehn \& Yaar stage & & $2.1(0.4)$ & $2.1(0.3)$ & & Mann-Whitney 0.835 & \\
\hline UPDRS-III & & $28.3(12.6)$ & $34.6(10.3)$ & & Mann-Whitney 0.057 & \\
\hline LEDD & & $586.7(304.8)$ & 748.5 (396.9) & 1906 & ANOVA 0.176 & \\
\hline MoCA & $26.6(1.5)$ & $26.5(1.5)$ & $20.9(1.5)$ & & Kruskal-Wallis $0.000 \mathbb{I}$ & 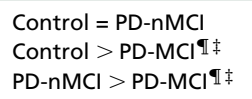 \\
\hline INECO & $22.8(2.5)$ & $20.8(2.4)$ & $16.6(3.5)$ & 26,281 & ANOVA $0.000 \mathbb{I}$ & 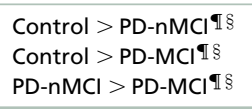 \\
\hline \multicolumn{7}{|c|}{$\begin{array}{l}\text { †ANOVA, distribution F. } \\
\text { ¥Mann-Whitney U test. } \\
\text { §Tukey test. } \\
\text { Ip }<0.05 ; \text { ' =': No significant difference; '>': Significantly higher than. } \\
\text { H\&Y: Hoehn \& Yahr stage; INECO: INECO frontal screening test; LEDD: Levodopa equivalent daily dose; MoCA: Montreal Cognitive Assessment; PD: Parkinson's disease; PD-nMCI: PD } \\
\text { without mild cognitive impairment; PD-MCI: PD with mild cognitive impairment; UPDRS: Unified Parkinson's Disease Rating Scale. }\end{array}$} \\
\hline
\end{tabular}

analysis was used; otherwise, nonparametric analysis was applied. In the same way, appropriate post-hoc tests were used to assess differences between groups (Table 1).

\section{Connectivity measures}

To distinguish variations in FC related to the base pathology from those associated to the occurrence of cognitive impairment, group comparisons were made in fixed pairs. Thus, analyses were performed comparing PD-nMCI versus controls, and PD-MCI versus PD-nMCI.

For every frequency band, differences in coherence were analyzed through a repeated measure mixed-design ANOVA performed for each connectivity type. As independent variables, group belonging was the intersubjects factor, and ROIs interactions within each connectivity type (levels) were the intrasubjects one. The GreenhouseGeisser correction of p-value was used when sphericity was not satisfied using the Mauchly's test.

Levels within each connectivity types were the repeated measures factor in the described ANOVA model. Hence, local coherence accounted for eight levels (bilateral frontal, temporal, parietal and occipital); intrahemispheric coherence held eight levels (frontotemporal, frontoparietal, temporo-occipital and parieto-occipital in both hemispheres); and interhemispheric coherence had four levels (interfrontal, intertemporal, interparietal and interoccipital).

For long-distance connectivity (intra and interhemispheric), post hoc analyses were performed by independent t-test comparisons by connectivity Levels if there was a significant main effect of Group, or an Interaction effect between Group and repeated measure factor. For short-distance coherence (IntraROI), differences between groups 
at each level of the intrasubjects factor were tested regardless the significance of the ANOVA main effects with the intention of examining topographical differences. As an exploratory study in a previously nondescribed population, we do not correct multiplicity for comparisons by ROIs interactions (levels) within each connectivity type.

\section{Correlations with executive function measure}

The IFS assess executive functions mostly reliant on frontal cortices. Thus, correlations between intrafrontal coherence and IFS scores were assessed in band frequencies in which PD groups had significant differences, using the Spearman's rank correlation coefficient.

All statistical analyses were performed at a significance level of 0.05 , using the SPSS software (version 23, IBM Corp. NY, USA).

\section{Results}

\section{Subject's characteristics}

There were not significant differences between the experimental groups regarding age, sex or educational level. PD-nMCI and PD-MCI were not different in disease progression, motor disability or levodopa equivalent daily dose. All the participants in our study, patients and controls, were right-handed. General features of groups and comparative statistics are shown in Table 1.

\section{Global cognition \& executive function}

MoCA test scores were significantly smaller for PD-MCI (mean $=20.9$; standard deviation $[S D]=1.5$ ) than for controls $($ mean $=26.6 ; \mathrm{SD}=1.5)$ and PD-nMCI $($ mean $=26.5 ; \mathrm{SD}=1.5)$ (Table 1). Relative to IFS, ANOVA indicated significant differences between study groups $(\mathrm{F}(2,69)=26.281 \mathrm{p}<0.001)$. Pairwise comparisons by post hoc Tukey procedure showed significant differences between all groups, with controls ( $m e a n=22.8, \mathrm{SD}=2.5$ ) scoring better than PD-nMCI (mean $=20.8, \mathrm{SD}=2.4)$, and PD-MCI ( mean = 16.6, SD = 3.5) scoring worse than controls and PD-nMCI (Table 1).

\section{Functional connectivity}

Intrahemispheric coherence

PD-nMCI versus controls

There was a significant main effect of Group in alfa2 band $(\mathrm{F}(1,56)=9.995, \mathrm{p}<0.003, \eta 2=15.1 \%)$. Post hoc comparisons between groups by ROI interactions showed that PD-nMCI patients had lower coherence than controls in bilateral fronto-temporal (left: $\mathrm{p}=0.003, \mathrm{~d}=-0.91$; right: $\mathrm{p}=0.018, \mathrm{~d}=-0.70$ ) and frontoparietal (left: $\mathrm{p}=0.014, \mathrm{~d}=-0.74$; right: $\mathrm{p}=0.018, \mathrm{~d}=-0.68)$ interplays. No other significant effects were found from ANOVA (Figure 2).

\section{PD-MCI versus PD-nMCI}

A main effect of Group was significant in alfa2 $\left(\mathrm{F}(1,34)=4.529, \mathrm{p}<0,05, \eta^{2}=11.8 \%\right)$. PD-MCI presented significant higher frontoparietal coherence than PD-nMCI group in both hemispheres (left: $p=0.022, d=0.74$; right: $\mathrm{p}=0.038, \mathrm{~d}=0.68$ ). No other significant effects were found (Figure 3 ).

\section{Interhemispheric coherence}

PD-nMCI versus controls

A significant Interaction effect between Group and ROIs interactions was found in alfa2 $(\mathrm{F}(3,168)=3.704$, $\left.\mathrm{p}<0.017, \eta^{2}=6.2 \%\right)$. PD-nMCI showed significant lower interparietal $(\mathrm{p}=0.025, \mathrm{~d}=-0.63)$ and interoccipital $(\mathrm{p}=0.025, \mathrm{~d}=-0.59)$ coherence than controls. There were not additional significant effects from the ANOVA (Figure 4A).

PD-MCI versus PD-nMCI

It was found a significant Interaction effect between Group and ROIs interactions in betal $(\mathrm{F}(3,102)=4.800$, $\left.\mathrm{p}<0.007, \eta^{2}=12.4 \%\right)$. PD-MCI presented lower interfrontal $(\mathrm{p}=0.017, \mathrm{~d}=-0.83)$, and higher interparietal $(\mathrm{p}=0.023, \mathrm{~d}=0.80)$ coherences, compared with PD-nMCI group. No other significant effect was found from the ANOVA (Figure 4B). 


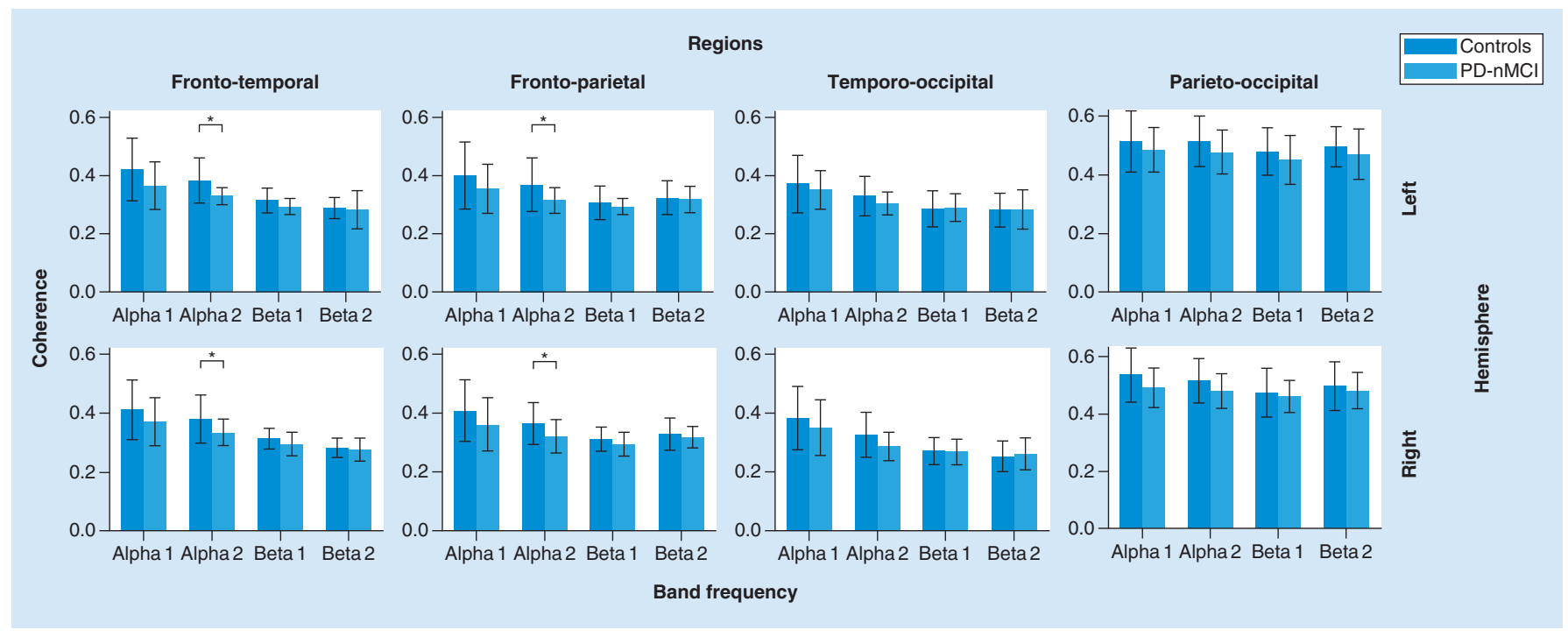

Figure 2. Intrahemispheric connectivity: comparisons between Parkinson's disease without mild cognitive impairment and controls. PD-nMCl: Parkinson's disease without mild cognitive impairment.

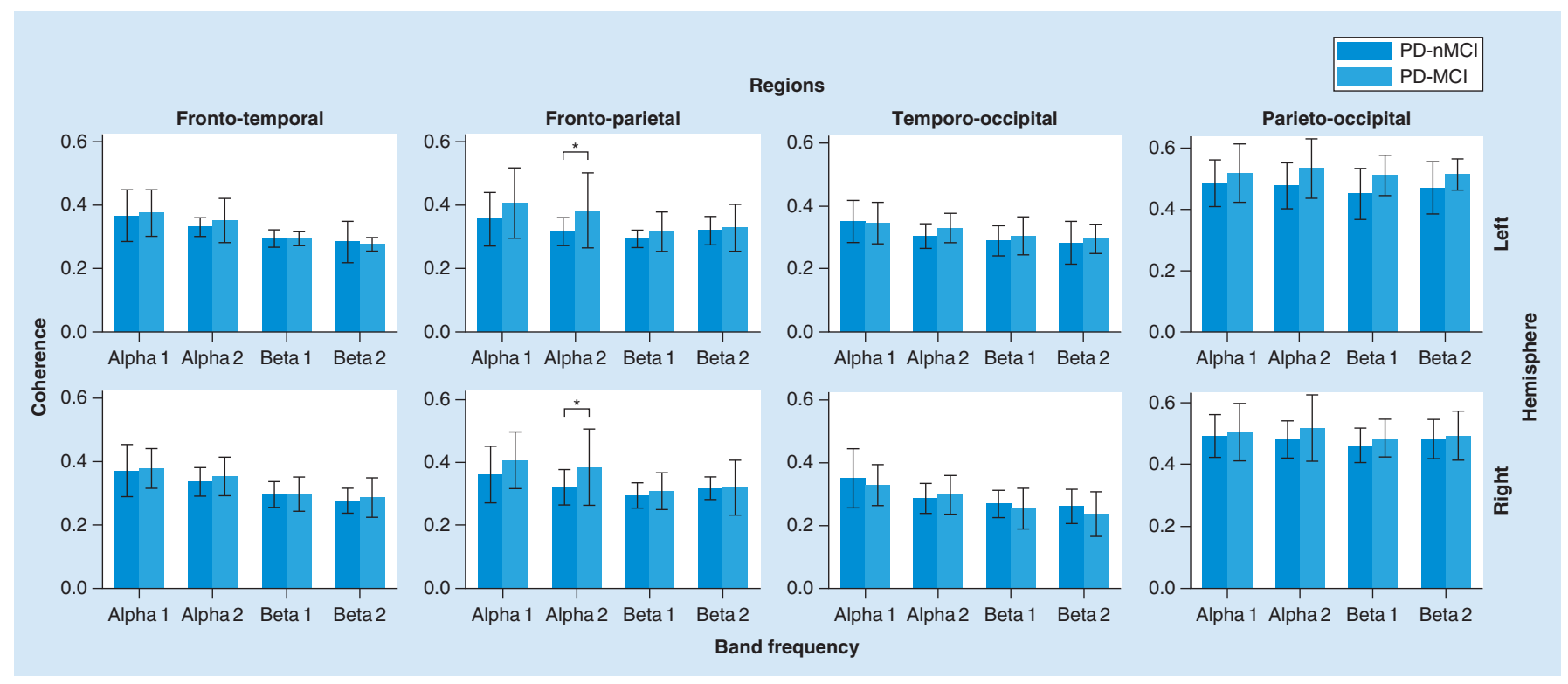

Figure 3. Intrahemispheric connectivity comparisons between Parkinson's disease with and without mild cognitive impairment. PD-nMCl: Parkinson's disease without mild cognitive impairment; PD-MCI: Parkinson's disease with mild cognitive impairment.

\section{Local coherence}

PD-nMCI versus controls

No significant main effects of Group or Interaction were observed from the repeated measure ANOVA. There were no differences in comparisons of group by ROIs interactions.

PD-MCI versus PD-nMCI

No significant main effects of Group or Interaction were observed from the ANOVA. However, there were significant differences regarding frontal ROIs between groups. In the left hemisphere, PD-MCI presented significant lower intrafrontal coherence in beta1 $(p=0.011, d=-0.87)$ and beta2 (left: $\mathrm{p}=0.012 \mathrm{~d}=-0.87$ ) bands (Figure 5). Right hemisphere presented the same trend. 


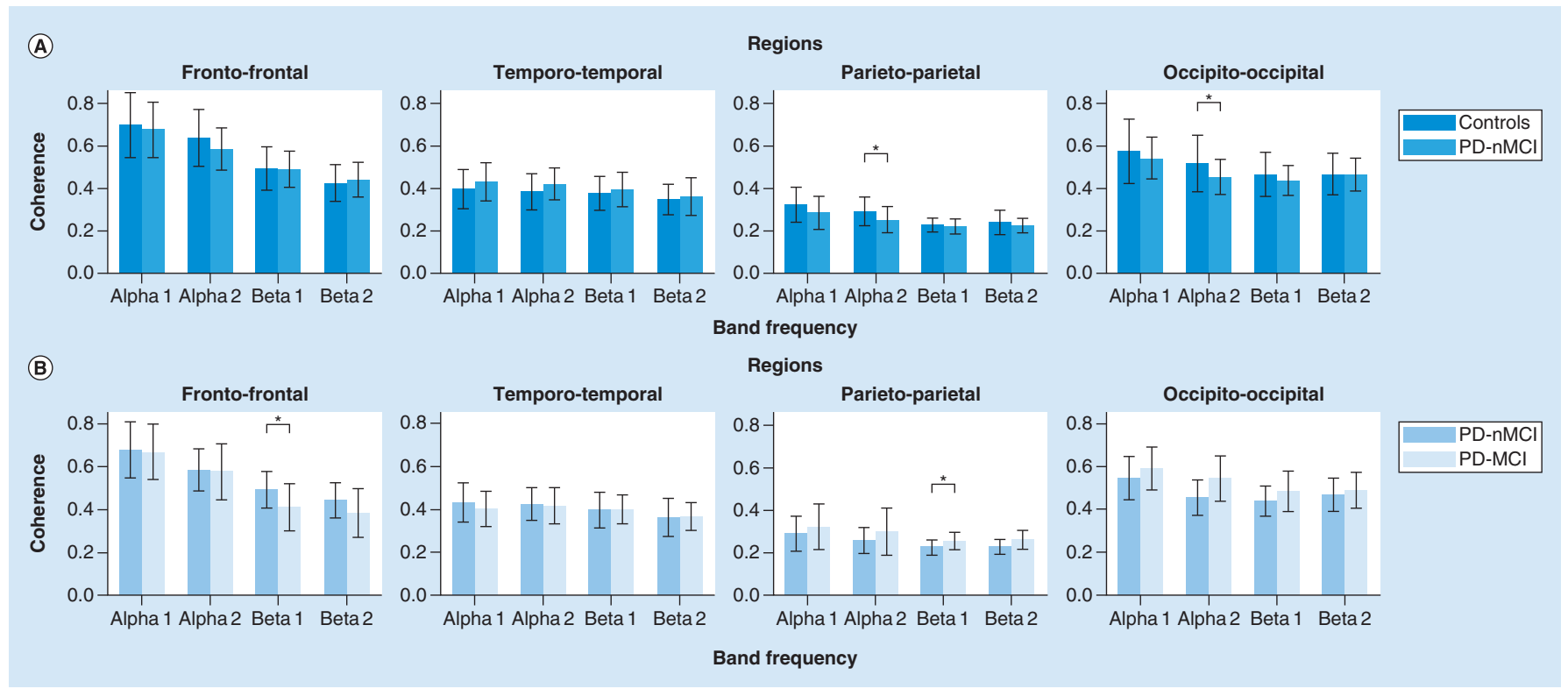

Figure 4. Interhemispheric connectivity results.

PD-nMCl: Parkinson's disease without mild cognitive impairment; PD-MCl: Parkinson's disease with mild cognitive impairment.

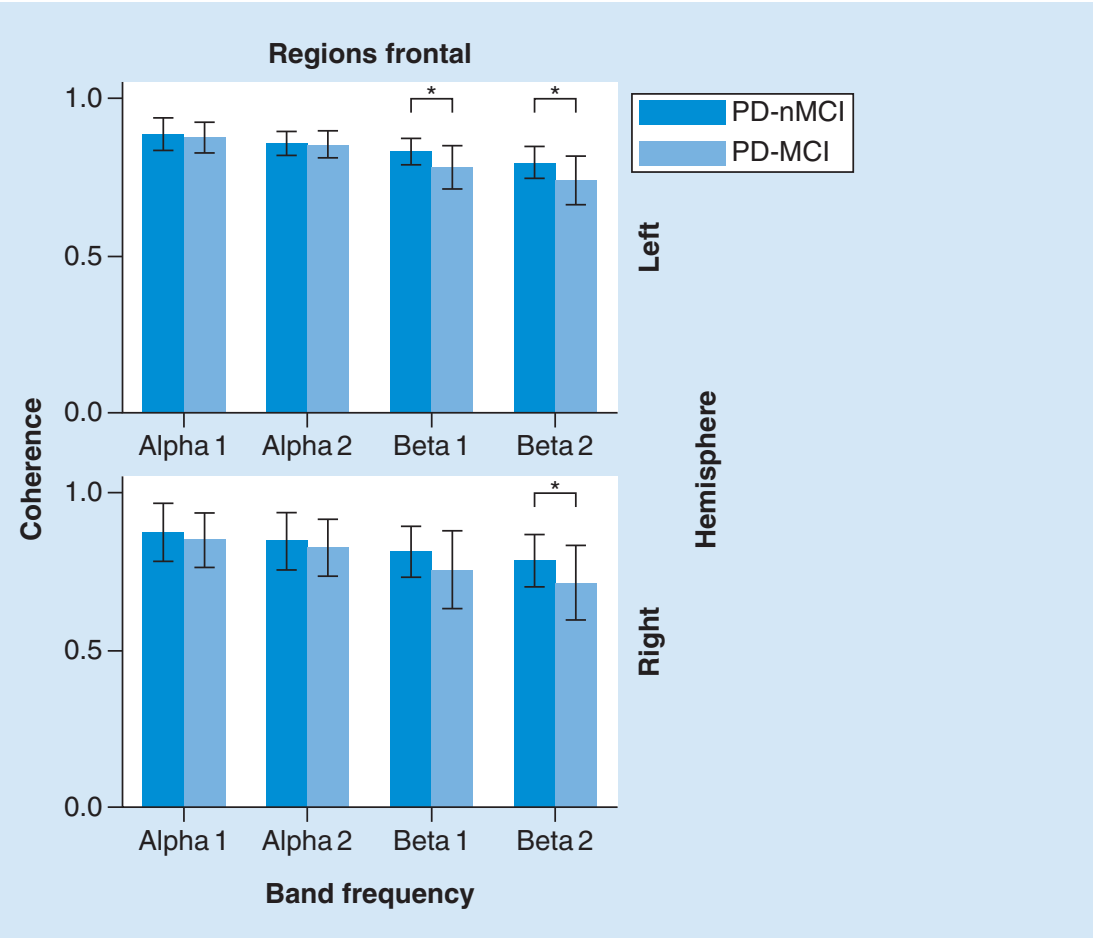

Figure 5. Intrafrontal connectivity in groups with Parkinson's disease.

PD-nMCI: Parkinson's disease without mild cognitive impairment; PD-MCI: Parkinson's disease with mild cognitive impairment.

\section{Correlations between frontal coherence \& executive functions}

There were significant correlations between IFS scores and intrafrontal coherence in patients. PD-nMCI group presented significant positive correlation in beta2 for right frontal ROI $(\mathrm{Rho}=0.539, \mathrm{p}=0.010)$. On the contrary, PD-MCI presented negative correlation in beta2 for left frontal ROI ( $R h o=-0.593, \mathrm{p}=0.025)$. 
It is important to note that UPDRS-III, LEDD and disease duration, had not significant correlations with intrafrontal coherence in the PD groups.

\section{Discussion}

The present study aimed to determinate the resting EEG FC features of a PD-ND population in relation to its cognitive status. We found a distinct FC pattern in PD-nMCI patients compared with PD-MCI and controls. Additionally, connectivity within frontal regions correlated differentially with executive functioning along PDND cognitive spectrum. Taken as a whole, our preliminary results of three types of connectivity reflect current pathophysiology hypothesis, clinical observations and recent empirical evidence on cognitive decline in PD.

Main results showed lower intrahemispheric (frontoparietal; frontotemporal) coherence in alfa2 in PD-nMCI, compared with controls. Conversely, PD-MCI presented higher intrahemispheric (frontoparietal) coherence in alfa2, in comparison with PD-nMCI. Previous EEG/MEG reports of resting connectivity in PD has pointed to an atypical synchronization involving mainly alfa and beta bands [43,45-47,55,56,85-87]. Particularly, PD-ND patients have shown regional differences with lower parietal coherence in alpha, and higher frontal coherence in beta frequencies in comparison to healthy controls [43].

Regarding our results, the major comparative limitation of the precedent evidence is that most of the EEG/MEG reports do not categorize PD patients with $\mathrm{MCI}$ as a separate group. Thus, previous EEG connectivity research would be displaying mixed cortical coupling patterns from PD-ND subpopulations with presumable distinct cortical dynamics [45,88-91]. Hence, the present study represents an advance addressing appropriate cognitive categorization of PD subjects without dementia in qEEG inquiry.

Other image techniques have studied PD individuals with MCI. Resting functional magnetic resonance images (fMRI) have revealed decreased connectivity of the right medial temporal lobe and bilateral inferior parietal cortex within the default mode network (DMN) in PD-nMCI, when compared with controls [92]. Structural connectivity studies have shown greater disturbances in frontal and anterior interhemispheric connections in PDMCI, in comparison to PD-nMCI [93,94]. Of particular interest, PD-MCI patients have shown mixed structural changes implicating gray matter thinning in prefrontal, insular and parietal regions [95-97] and thickening in right frontoparietal cortices, if contrasted to PD-nMCI [95]. Additionally, PD-MCI individuals have shown increased metabolic signals in cingulate areas at rest [98], and parietal cortices during cognitive tasks [99].

A growing amount of evidence indicate that connectivity modifications in PD-ND depend on cognitive status of patients $[42,100]$. Our findings suggest also that when global cognitive decay is clinically identifiable, as in PD-MCI, an adaptive/reactive cortical activity would become evident concerning intrahemispheric interactions, possibly in an attempt to maintain general efficiency of cognitive networks [35,90,101].

As a secondary central finding, we found lower posterior interhemispheric (interparietal) coherence in alpha2 for PD-nMCI when contrasted with controls. Meanwhile, PD-MCI showed higher interparietal coherence in beta1, compared with PD-nMCI. In this direction, previous electrophysiological [85], structural [95,96,102,103] and functional image studies [92], has described shifting changes in posterior cortices associated to cognitive impairment in PD. In particular, PD-MCI subjects have shown a dissociation in FC between anterior and posterior brain regions with respect to Intrinsic Connectivity Networks in resting fMRI [104]. Specifically, PD-MCI presented reduced connectivity between right fronto-insular areas and dorsal attentional network (DAN), together with enhanced connectivity between parieto-occipital areas and DMN components [104].

In the same regard, structural studies have described a pattern of connectivity irregularities across the cognitive spectrum of PD [105], involving anterior tracts initially [106,107], with further implication of posterior connection fibers [102,108]. Altogether, changes in EEG connectivity in PD-MCI found in our study, appear to be driven by adaptive/reactive activities concerning posterior cortices [98,99,109,110], suggesting a compensatory (or deregulated) interhemispheric synchronization through alfa2-beta1 range. This enhanced cortical coupling in PD-MCI could be reflecting recruitments of additional processing resources reliant on the less affected posterior regions [111], to sustain global functionality of cognitive networks [90].

A third central outcome revealed reductions of intra and interfrontal coherence in the beta range for PD-MCI, compared with PD-nMCI. Other resting and task related functional studies have reported progressive frontal alterations related to cognitive decline in PD. In this way, in comparison to PD-nMCI, subjects with PD-MCI have presented decreased FC in the striatum [112], and in frontal [90], prefrontal [113] and fronto-insular regions [104], within DMN, frontoparietal network and DAN, respectively. 
In this direction, PD-MCI patients have shown striatal dopamine depletion and reduced receptor (D2) availability in the anterior insula [114]. The anterior fronto-insular area (salience network) seems to play a critical role in switching between central executive network (dorsolateral prefrontal, parietal cortices) and DMN (medial prefrontal, posterior parietal) [29,115], which normal interaction has been linked to an appropriate executive functioning [29,116]. Overall, our findings support the hypothesis that a progressive fronto-striatal dysfunction underlies early cognitive impairment in PD, particularly for executive functions $[104,113,117,118]$.

Finally, patients shown differential significant correlations in frontal ROIs in beta 2 band. Specifically, PD- nMCI had positive correlation on the right hemisphere, whereas PD-MCI exhibits a negative correlation on the left one. In the same direction, a recent EEG resting study found significant correlations between frontoparietal coherence in conventional fast frequencies and executive dysfunctions in PD-ND, but MCI condition was not analyzed separately [56]. Furthermore, several fMRI studies at rest [94], and others during cognitive tasks [99,112,119], have demonstrated correlations between anomalous activity in frontostriatal regions and a dysexecutive profile in PD without dementia. In effect, alterations in dopamine-dependent activity within the striatum [114,120,121], insular and prefrontal regions [122-124], have shown to be associated directly to executive performance in PD.

The aforementioned results are in accordance with reports showing that PD-MCI had further underactivity (decreased BOLD signal) during executive related tasks, in right caudate, right prefrontal dorsolateral and anterior cingulate areas, when compared with PD- nMCI [99,112]. These observations suggest that activity modulations in frontal cortices induced by striatal dopamine depletion in $\mathrm{PD}$, may contribute to unusual interactions with other cortical components of networks involved in executive processing [35,36].

Interestingly, our analysis of correlations indicated a possible role of the right prefrontal region in maintaining executive and global cognition efficiency in the PD-nMCI individuals, in relation to synchronization activity in beta frequencies. This assertion have support in the proposed lateralization of executive functions in PD [125,126], in the fact that it has been also described in cognitively unaffected aged individuals [127], and in EEG observations of positive correlations between executive functioning and frontal coherence in beta in nondemented healthy older adults [128]. According to this approach, we speculate that the right prefrontal regions would be important in preserving global cognition in PD-nMCI based on right hemisphere functional attributes such as monitoring, attentional and visuospatial processing [127,129]. This is a plausible association because deficits in some of these functions are associated with the transition to a more advanced cognitive deterioration [13], following closely the dual syndrome hypothesis of PD [22].

\section{Limitations}

This study has some limitations. First, we used the MoCA as a global cognition measure to classify patients, reaching level I of PD-MCI criteria [72], and thus entailing less diagnostic certainty. Second, patients were evaluated in phase 'On' of their PD medication. Thereby, the confounding influence of long-standing dopaminergic therapy cannot be appropriately assessed. Nevertheless, data analysis did not show significant correlations between LEDD and the coherence patterns we found. Third, sample size is relatively small, especially for PD-MCI. Hence, general outcomes could not balance sufficiently variability between subjects. Further EEG studies are required to verify present findings in larger cohorts of PD-MCI patients, and to appraise sensitivity and specificity of FC markers. Fourth, coherence between near electrodes could be due to a volume conductance effect, generating spurious correlations in the EEG signal space.

The last aspect deserve an extension of discussion. In the first place, the contribution of the volume conductance on coherence could be assessed as a function of distance between electrodes. Coherence due to volume conductance have the tendency to approximate zero in a critical distance between 5 and $17 \mathrm{~cm}[130,131]$. Considering the average head circumference of our participants $(57.1 \mathrm{~cm}$ ), and that all midline electrodes was excluded from analysis, we calculated a mean of the minimum interhemispheric electrode separation of $5.3 \mathrm{~cm}$, which would be in the close-to-zero range of coherence due to volume conductance. Therefore, given that our main findings involved long-distance interactions, it is less likely that our results could be extensively contaminated by volume conductance.

One solution to the volume conductance problem is to estimate coherence applying source reconstruction methods [84,132,133]. Nevertheless, there is not a unique methodology to localize sources [133], and some techniques to solution the inverse problem are not compatible with certain connectivity measures [134]. Thus, we choose a pragmatic approach, focusing the analysis in the signal space. This way, even if the volume conductance could affect coherence, it is unlikely that it can explain the confluence of outcomes in the two arranges of group comparisons, the 
consistency of findings in different types of connectivity, and the significant differences in long-distance connectivity between groups of patients with different cognitive profile.

Noteworthy, general results of this study are in general accord with current pathophysiology hypothesis and clinical observations [22], and share similar directions in findings registered with other brain-based techniques [100]. This allows us to assume that connectivity measures we estimated in the present study correspond more with coherence between genuine neural sources, that with a steady volume conductance influencing evenly the majority of subjects. Nevertheless, future EEG studies assessing comparable connectivity measures, electrode assortments and precise source localizations of EEG sources are warranted.

\section{Conclusion}

The current study demonstrates a compelling relationship between EEG FC patterns during resting wakefulness and the cognitive status in a nondemented PD population. Decrements in intrahemispheric coherence in alfa2 could be an early EEG marker of the primary pathology with respect to normal elderly subjects. Conversely, increases in intrahemispheric coherence in alpha2, and posterior interhemispheric coherence in alpha2-beta1 range, might be the correlates of transition to global cognitive decline. All things considered, outcomes presented in this study follow general postulates of the dual syndrome hypothesis in PD [13,18,22,135], supporting the premise that progressive prefrontal dysfunction - caused by striatal dopamine depletion - may influence large-scale networks activity, interfering with specific cognitive processes.

Overall, because of the direct sensibility to neuronal activity, the greater time resolution, relative low costs, noninvasiveness and the little collaboration needed from subjects, the resting-state EEG connectivity analysis can be useful as a biomarker to assist diagnosis, interventions and/or cognitive prognosis in PD.

\section{Future perspective}

In subsequent years, the clinical use of sensitive and accurate diagnostic tools with capacity to track significant brain dysfunctions will be used to early detect disorders that could lead to dementia. The need for cheap, practical and widely available diagnostic biological markers will lead to a greater use of neurophysiologic techniques such as the qEEG.

Research and clinical applications of qEEG analysis in different neurodegenerative disorders will allow scientific community to identify relevant qEEG measures and proper processing algorithms to be reliably used in the assistance of individual patients.

\section{Financial \& competing interests disclosure}

This study was supported by the Research Development Committee (CODI) of the University of Antioquia, Colombia. Research project: "Procesamiento del lenguaje de acción en pacientes con enfermedad ganglio-basal desde una perspectiva neuropsicológica y neurofisiológica" (grant reference PRG-2014-768). The study sponsor had no role in the collection, analysis or interpretation of data, nor in the writing of the final manuscript. The authors have no other relevant affiliations or financial involvement with any organization or entity with a financial interest in or financial conflict with the subject matter or materials discussed in the manuscript. This includes employment, consultancies, honoraria, stock ownership or options, expert testimony, grants or patents received or pending, or royalties.

No writing assistance was utilized in the production of this manuscript.

Ethical disclosure

The authors state that the present study protocol had the approval of the Ethical Research Committee of the University of Antioquia (No. 15-10-569). All participants gave their written informed consent after an explanation of the study characteristics, and before any clinical or experimental evaluation, according to the declaration of Helsinki.

\section{Author contributions}

Please list the contributions of each author to the piece; please refer to the Author Disclosure Form for our authorship criteria.

\section{Open access}

This work is licensed under the Creative Commons Attribution-NonCommercial-NoDerivatives 4.0 Unported License. To view a copy of this license, visit: http://creativecommons.org/licenses/by-nc-nd/4.0/ 


\section{Summary points}

Cognitive impairment in Parkinson's disease

- Cognitive disturbances are frequent in Parkinson's disease (PD) and mild cognitive impairment (MCI) is recognized as an important risk factor for developing dementia in the course of disease.

Brain networks \& cognition

- Pathophysiology of cognitive impairment in PD entails cortical connectivity disruptions affecting global functionality of organized circuits.

Electrophysiology \& cognitive states

- Neurophysiological correlates of cognitive states can be tracked through practical noninvasive brain-based techniques such as the electroencephalogram (EEG) signals.

Functional connectivity approach

- Functional connectivity (FC) is the analytical approach that examines statistical dependencies between brain signals originated from different neural units.

EEG connectivity in PD

- EEG connectivity studies in PD without dementia (PD-ND) have shown that FC measures might have the ability to distinguish patients from healthy age-matched subjects.

- There is a lack of evidence about relations between EEG FC measures and the cognitive profile of patients.

Study methods

- We compared EEG coherence in two groups of PD-ND subjects with $\mathrm{MCl}$ (PD-MCI) and without $\mathrm{MCl}$ (PD-nMCI).

Global results

- There were differences in intra and interhemispheric coherence between PD-nMCl and PD-MCI. FC disparities appears over alpha2-beta1 band frequency width.

- We observed correlations between intrafrontal connectivity in beta2 and global executive functioning in PD patients.

Conclusion

- EEG coherence measures indicate distinct resting-state cortical activity in PD subjects with and without $\mathrm{MCl}$.

- Integrated results are in accord with current pathophysiology hypothesis, clinical observations and recent neuroimaging findings.

- This study suggests that the EEG connectivity analysis is a potential biomarker to monitor cognitive disturbances in PD.

\section{References}

1. de Lau LML, Breteler MMB. Epidemiology of Parkinson's disease. Lancet. Neurol. 5(6), 525-535 (2006).

2. Kalia LV, Lang AE. Parkinson's disease. Lancet 386(9996), 896-912 (2015).

3. Jankovic J. Parkinson's disease: clinical features and diagnosis. J. Neurol. Neurosurg. Psychiatry 79(4), 368-376 (2008).

4. Martinez-Martin P, Rodriguez-Blazquez C, Kurtis MM, Chaudhuri KR. The impact of non-motor symptoms on health-related quality of life of patients with Parkinson's disease. Mov. Disord. 26(3), 399-406 (2011).

5. Khoo TK, Yarnall AJ, Duncan GW et al. The spectrum of nonmotor symptoms in early Parkinson disease. Neurology 80(3), 276-281 (2013).

6. Duncan GW, Khoo TK, Yarnall AJ et al. Health-related quality of life in early Parkinson's disease: the impact of nonmotor symptoms. Mov. Disord. 29(2), 195-202 (2014).

7. Yang Y, Tang B-S, Guo J-F. Parkinson's disease and cognitive impairment. Parkinsons Dis. 2016, 6734678 (2016).

8. Santangelo G, Vitale C, Picillo M et al. Mild cognitive impairment in newly diagnosed Parkinson's disease: a longitudinal prospective study. Parkinsonism Relat. Disord. 21(10), 1219-1226 (2015).

9. Yarnall AJ, Breen DP, Duncan GW et al. Characterizing mild cognitive impairment in incident Parkinson disease: the ICICLE-PD study. Neurology 82(4), 308-316 (2014).

10. Aarsland D, Bronnick K, Larsen JP, Tysnes OB, Alves G. Cognitive impairment in incident, untreated Parkinson disease: the Norwegian ParkWest study. Neurology 72(13), 1121-1126 (2009).

11. Aarsland D, Zaccai J, Brayne C. A systematic review of prevalence studies of dementia in Parkinson's disease. Mov. Disord. 20(10), 1255-1263 (2005).

12. Muslimovic D, Post B, Speelman JD, Schmand B. Cognitive profile of patients with newly diagnosed Parkinson disease. Neurology 65(8), 1239-1245 (2005).

13. Pagonabarraga J, Kulisevsky J. Cognitive impairment and dementia in Parkinson's disease. Neurobiol. Dis. 46(3), 590-596 (2012). 
14. Broeders M, de Bie RMA, Velseboer DC, Speelman JD, Muslimovic D, Schmand B. Evolution of mild cognitive impairment in Parkinson disease. Neurology 81(4), 346-352 (2013).

15. Monchi O, Hanganu A, Bellec P. Markers of cognitive decline in PD: the case for heterogeneity. Parkinsonism Relat. Disord. 24, 8-14 (2016).

16. Bocanegra Y, Trujillo-Orrego N, Pineda D. [Dementia and mild cognitive impairment in Parkinson's disease: a review]. Rev. Neurol. 59(12), 555-569 (2014).

17. Robbins TW, Cools R. Cognitive deficits in Parkinson's disease: a cognitive neuroscience perspective. Mov. Disord. 29(5), 597-607 (2014).

18. Kehagia AA, Barker RA, Robbins TW. Neuropsychological and clinical heterogeneity of cognitive impairment and dementia in patients with Parkinson's disease. Lancet. Neurol. 9(12), 1200-1213 (2010).

19. Domellof ME, Ekman U, Forsgren L, Elgh E. Cognitive function in the early phase of Parkinson's disease, a five-year follow-up. Acta Neurol. Scand. 132(2), 79-88 (2015).

20. Caviness JN, Driver-Dunckley E, Connor DJ et al. Defining mild cognitive impairment in Parkinson's disease. Mov. Disord. 22(9), 1272-1277 (2007).

21. Hobson P, Meara J. Mild cognitive impairment in Parkinson's disease and its progression onto dementia: a 16-year outcome evaluation of the Denbighshire cohort. Int. J. Geriatr. Psychiatry 30(10), 1048-1055 (2015).

22. Kehagia AA, Barker RA, Robbins TW. Cognitive impairment in Parkinson's disease: the dual syndrome hypothesis. Neurodegener. Dis. 11(2), 79-92 (2013).

23. Hilker R, Thomas AV, Klein JC et al. Dementia in Parkinson disease: functional imaging of cholinergic and dopaminergic pathways. Neurology 65(11), 1716-1722 (2005).

24. Bonelli RM, Cummings JL. Frontal-subcortical circuitry and behavior. Dialog. Clin. Neurosci. 9(2), 141-151 (2007).

25. DeLong MR, Wichmann T. Circuits and circuit disorders of the basal ganglia. Arch. Neurol. 64(1), 20-24 (2007).

26. Middleton FA, Strick PL. Basal ganglia output and cognition: evidence from anatomical, behavioral, and clinical studies. Brain Cogn 42(2), 183-200 (2000).

27. Sporns O, Tononi G, Edelman GM. Connectivity and complexity: the relationship between neuroanatomy and brain dynamics. Neural Netw. 13(8-9), 909-922 (2000).

28. Varela F, Lachaux JP, Rodriguez E, Martinerie J. The brainweb: phase synchronization and large-scale integration. Nat. Rev. Neurosci. 2(4), 229-239 (2001).

29. Bressler SL, Menon V. Large-scale brain networks in cognition: emerging methods and principles. Trends Cogn. Sci. 14(6), 277-290 (2010).

30. Fietta P, Fietta P. Cognition: neurobiological correlates and dynamics. Theor. Biol. Forum. 105(1), 87-108 (2012).

31. Pfurtscheller G, Lopes da Silva FH. Event-related EEG/MEG synchronization and desynchronization: basic principles. Clin. Neurophysiol. 110(11), 1842-1857 (1999).

32. Pereda E, Quiroga RQ, Bhattacharya J. Nonlinear multivariate analysis of neurophysiological signals. Prog. Neurobiol. 77(1-2), 1-37 (2005).

33. Sakkalis V. Review of advanced techniques for the estimation of brain connectivity measured with EEG/MEG. Comput. Biol. Med. 41(12), 1110-1117 (2011).

34. Park H-J, Friston K. Structural and functional brain networks: from connections to cognition. Science 342(6158), 1238411 (2013).

35. Caviness JN. Pathophysiology of Parkinson's disease behavior-a view from the network. Parkinsonism Relat. Disord. 20(Suppl. 1), S39-43 (2014).

36. Gratwicke J, Jahanshahi M, Foltynie T. Parkinson's disease dementia: a neural networks perspective. Brain 138(Pt 6), 1454-1476 (2015).

37. Babiloni C, Lizio R, Marzano N et al. Brain neural synchronization and functional coupling in Alzheimer's disease as revealed by resting state EEG rhythms. Int. J. Psychophysiol. 103, 88-102 (2016).

38. Carlino E, Sigaudo M, Rosato R, Vighetti S, Rocca P. Electroencephalographic connectivity analysis in schizophrenia. Neurosci. Lett. 604, 145-150 (2015).

39. Olbrich S, Olbrich H, Adamaszek M, Jahn I, Hegerl U, Stengler K. Altered EEG lagged coherence during rest in obsessive-compulsive disorder. Clin. Neurophysiol. 124(12), 2421-2430 (2013).

40. Cover KS, Vrenken H, Geurts JJG et al. Multiple sclerosis patients show a highly significant decrease in alpha band interhemispheric synchronization measured using MEG. Neuroimage. 29(3), 783-788 (2006).

41. Cerasa A, Novellino F, Quattrone A. Connectivity Changes in Parkinson's Disease. Curr. Neurol. Neurosci. Rep. 16(10), 91 (2016).

42. Gao L-L, Wu T. The study of brain functional connectivity in Parkinson's disease. Transl. Neurodegener. 5, 18 (2016).

43. Moazami-Goudarzi M, Sarnthein J, Michels L, Moukhtieva R, Jeanmonod D. Enhanced frontal low and high frequency power and synchronization in the resting EEG of parkinsonian patients. Neuroimage. 41(3), 985-997 (2008). 
44. Pugnetti L, Baglio F, Farina E et al. EEG evidence of posterior cortical disconnection in PD and related dementias. Int. J. Neurosci. 120(2), 88-98 (2010).

45. Stoffers D, Bosboom JLW, Deijen JB, Wolters EC, Stam CJ, Berendse HW. Increased cortico-cortical functional connectivity in early-stage Parkinson's disease: an MEG study. Neuroimage 41(2), 212-222 (2008).

46. Silberstein P, Pogosyan A, Kuhn AA et al. Cortico-cortical coupling in Parkinson's disease and its modulation by therapy. Brain $128(\mathrm{Pt}$ 6), 1277-1291 (2005).

47. George JS, Strunk J, Mak-McCully R, Houser M, Poizner H, Aron AR. Dopaminergic therapy in Parkinson's disease decreases cortical beta band coherence in the resting state and increases cortical beta band power during executive control. NeuroImage Clin. 3, 261-270 (2013).

48. Stoffers D, Bosboom JLW, Wolters EC, Stam CJ, Berendse HW. Dopaminergic modulation of cortico-cortical functional connectivity in Parkinson's disease: an MEG study. Exp. Neurol. 213(1), 191-195 (2008).

49. Nuwer M. Assessment of digital EEG, quantitative EEG, and EEG brain mapping: report of the American Academy of Neurology and the American Clinical Neurophysiology Society. Neurology 49(1), 277-292 (1997).

50. de Medeiros Kanda PA, Anghinah R, Smidth MT, Silva JM. The clinical use of quantitative EEG in cognitive disorders. Dement. Neuropsychol. 3, 195-203 (2009).

51. Locatelli T, Cursi M, Liberati D, Franceschi M, Comi G. EEG coherence in Alzheimer's disease. Electroencephalogr. Clin. Neurophysiol. 106(3), 229-237 (1998).

52. Czigler B, Csikos D, Hidasi Z et al. Quantitative EEG in early Alzheimer's disease patients - power spectrum and complexity features. Int. J. Psychophysiol. 68(1), 75-80 (2008).

53. Bonanni L, Thomas A, Tiraboschi P et al. EEG comparisons in early Alzheimer's disease, dementia with Lewy bodies and Parkinson's disease with dementia patients with a 2-year follow-up. Brain 131(3), 690-705 (2008).

54. Klassen BT, Hentz JG, Shill HA et al. Quantitative EEG as a predictive biomarker for Parkinson disease dementia. Neurology 77(2), 118-124 (2011).

55. Fonseca L, Tedrus G, Carvas P, Machado E. Comparison of quantitative EEG between patients with Alzheimer's disease and those with Parkinson's disease dementia. Clin. Neurophysiol. 124(10), 1970-1974 (2013).

56. Teramoto H, Morita A, Ninomiya S, Akimoto T, Shiota H, Kamei S. Relation between Resting State Front-Parietal EEG Coherence and Executive Function in Parkinson's Disease. Biomed Res. Int. 2016, 2845754 (2016).

57. Carmona J, Tobón C, Pineda D. Electroencephalographic markers and cognitive fenotype in Parkinson's disease. A systematic review. [Marcadores electroencefalográficos y fenotipo cognitivo en la enfermedad de Parkinson. Una revisión sistemática]40(3), 332-348 (2018).

58. Cozac VV, Gschwandtner U, Hatz F, Hardmeier M, Ruegg S, Fuhr P. Quantitative EEG and Cognitive Decline in Parkinson's Disease. Parkinsons. Dis. 2016, 9060649 (2016).

59. Geraedts VJ, Boon LI, Marinus J et al. Clinical correlates of quantitative EEG in Parkinson disease: a systematic review. Neurology 91(19), 871-883 (2018).

60. American Psychiatric Association. DSM-IV-TR: diagnostic and statistical manual of mental disorders. American Psychiatric Association (2000). http://www.worldcat.org/isbn/9780890420256

61. Gibb WR. Accuracy in the clinical diagnosis of parkinsonian syndromes. Postgrad. Med. J. 64(751), 345-351 (1988).

62. Hoehn MM, Yahr MD. Parkinsonism: onset, progression and mortality. Neurology 17(5), 427-442 (1967).

63. Goetz CG, Poewe W, Rascol O et al. Movement Disorder Society Task Force report on the Hoehn and Yahr staging scale: status and recommendations. Mov. Disord. 19(9), 1020-1028 (2004).

64. Goetz CG, Tilley BC, Shaftman SR et al. Movement Disorder Society-sponsored revision of the Unified Parkinson's Disease Rating Scale (MDS-UPDRS): scale presentation and clinimetric testing results. Mov. Disord. 23(15), 2129-2170 (2008).

65. Nasreddine ZS, Phillips NA, Bedirian V et al. The Montreal Cognitive Assessment, MoCA: a brief screening tool for mild cognitive impairment. J. Am. Geriatr. Soc. 53(4), 695-699 (2005).

66. Dalrymple-Alford JC, MacAskill MR, Nakas CT et al. The MoCA: well-suited screen for cognitive impairment in Parkinson disease. Neurology. 75(19), 1717-1725 (2010).

67. Kandiah N, Zhang A, Cenina AR, Au WL, Nadkarni N, Tan LC. Montreal Cognitive Assessment for the screening and prediction of cognitive decline in early Parkinson’s disease. Parkinsonism Relat. Disord. 20(11), 1145-1148 (2014).

68. Gil L, Ruiz de Sanchez C, Gil F, Romero SJ, Pretelt Burgos F. Validation of the Montreal Cognitive Assessment (MoCA) in Spanish as a screening tool for mild cognitive impairment and mild dementia in patients over 65 years old in Bogota, Colombia. Int. J. Geriatr. Psychiatry 30(6), 655-662 (2015).

69. Mahoney F, Barthel D. Functional Evaluation: The Barthel Index. Md State Med. J. 14, 61-65 (1965).

70. Lawton MP, Brody EM. Assessment of older people: self-maintaining and instrumental activities of daily living. Gerontologist $9(3)$, 179-186 (1969). 
71. Tomlinson CL, Stowe R, Patel S, Rick C, Gray R, Clarke CE. Systematic review of levodopa dose equivalency reporting in Parkinson's disease. Mov. Disord. 25(15), 2649-2653 (2010).

72. Litvan I, Goldman JG, Troster AI et al. Diagnostic criteria for mild cognitive impairment in Parkinson's disease: Movement Disorder Society Task Force guidelines. Mov. Disord. 27(3), 349-356 (2012).

73. Torralva T, Roca M, Gleichgerrcht E, Lopez P, Manes F. INECO Frontal Screening (IFS): a brief, sensitive, and specific tool to assess executive functions in dementia. J. Int. Neuropsychol. Soc. 15(5), 777-786 (2009).

74. Gleichgerrcht E, Roca M, Manes F, Torralva T. Comparing the clinical usefulness of the Institute of Cognitive Neurology (INECO) Frontal Screening (IFS) and the Frontal Assessment Battery (FAB) in frontotemporal dementia. J. Clin. Exp. Neuropsychol. 33(9), 997-1004 (2011).

75. Jurado MB, Rosselli M. The elusive nature of executive functions: a review of our current understanding. Neuropsychol. Rev. 17(3), 213-233 (2007).Suarez-Revelo J, Ochoa-Gomez J, Duque-Grajales J. Improving test-retest reliability of quantitative electroencephalography using different preprocessing approaches. Presented at: 38th Annual International Conference of the IEEE Engineering inMed icine and Biology Society FL, USA, 2016, 961-964 (2016).

77. Delorme A, Makeig S. EEGLAB: an open source toolbox for analysis of single-trial EEG dynamics including independent component analysis. J. Neurosci. Methods 134(1), 9-21 (2004).

78. Bigdely-Shamlo N, Mullen T, Kothe C, Su K-M, Robbins KA. The PREP pipeline: standardized preprocessing for large-scale EEG analysis. Front. Neuroinform. 9, 16 (2015).

79. Castellanos NP, Makarov VA. Recovering EEG brain signals: artifact suppression with wavelet enhanced independent component analysis. J. Neurosci. Methods 158(2), 300-312 (2006).

80. Delorme A, Sejnowski T, Makeig S. Enhanced detection of artifacts in EEG data using higher-order statistics and independent component analysis. Neuroimage 34(4), 1443-1449 (2007).

81. Mitra P, Hemant B. Observed Brain Dynamics. Oxford University Press, Inc., NY, USA (2007).

82. Mitra P, Bokil H, Maniar H et al. Chronux (2016). http://chronux.org/

83. Stam CJ, van Straaten ECW. The organization of physiological brain networks. Clin. Neurophysiol. 123(6), 1067-1087 (2012).

84. Bowyer SM. Coherence a measure of the brain networks: past and present. Neuropsychiatr. Electrophysiol.2(1), 1 (2016).

85. Bosboom JLW, Stoffers D, Wolters EC, Stam CJ, Berendse HW. MEG resting state functional connectivity in Parkinson's disease related dementia. J. Neural Transm. 116(2), 193-202 (2009).

86. Hassana M, Chaton L, Benquet P et al. Functional connectivity disruptions correlate with cognitive phenotypes in Parkinson's disease. NeuroImage Clin.14, 591-601 (2017).

87. He X, Zhang Y, Chen J et al. The Patterns of EEG Changes in early-onset Parkinson's disease patients. Int. J. Neurosci. 127(11), 1028-1035 (2017).

88. Caviness JN, Hentz JG, Evidente VG et al. Both early and late cognitive dysfunction affects the electroencephalogram in Parkinson's disease. Park. Relat. Disord.13(6), 348-354 (2007).

89. Fonseca L, Tedrus G, Letro G, Bossoni A. Dementia, mild cognitive impairment and quantitative EEG in patients with Parkinson's disease. Clin. EEG Neurosci. 40(3), 168-172 (2009).

90. Gorges M, Muller H-P, Lule D, Pinkhardt EH, Ludolph AC, Kassubek J. To rise and to fall: functional connectivity in cognitively normal and cognitively impaired patients with Parkinson's disease. Neurobiol. Aging 36(4), 1727-1735 (2015).

91. Utianski RL, Caviness JN, van Straaten ECW et al. Graph theory network function in Parkinson's disease assessed with electroencephalography. Clin. Neurophysiol. 127(5), 2228-2236 (2016).

92. Tessitore A, Esposito F, Vitale C et al. Default-mode network connectivity in cognitively unimpaired patients with Parkinson disease. Neurology 79(23), 2226-2232 (2012).

93. Melzer TR, Watts R, MacAskill MR et al. White matter microstructure deteriorates across cognitive stages in Parkinson disease. Neurology 80(20), 1841-1849 (2013).

94. Agosta F, Canu E, Stefanova E et al. Mild cognitive impairment in Parkinson's disease is associated with a distributed pattern of brain white matter damage. Hum. Brain Mapp. 35(5), 1921-1929 (2014).

95. Biundo R, Calabrese M, Weis L et al. Anatomical correlates of cognitive functions in early Parkinson's disease patients. PLoS ONE 8(5), e64222 (2013).

96. Melzer TR, Watts R, MacAskill MR et al. Grey matter atrophy in cognitively impaired Parkinson's disease. J. Neurol. Neurosurg. Psychiatry 83(2), 188-194 (2012).

97. Lee JE, Cho KH, Song SK et al. Exploratory analysis of neuropsychological and neuroanatomical correlates of progressive mild cognitive impairment in Parkinson's disease. J. Neurol. Neurosurg. Psychiatry 85(1), 7-16 (2014).

98. Huang C, Mattis P, Perrine K, Brown N, Dhawan V, Eidelberg D. Metabolic abnormalities associated with mild cognitive impairment in Parkinson disease. Neurology $70(16$ Pt 2), 1470-1477 (2008). 
99. Ekman U, Eriksson J, Forsgren L, Mo SJ, Riklund K, Nyberg L. Functional brain activity and presynaptic dopamine uptake in patients with Parkinson's disease and mild cognitive impairment: a cross-sectional study. Lancet. Neurol. 11(8), 679-687 (2012).

100. Biundo R, Weis L, Antonini A. Cognitive decline in Parkinson's disease: the complex picture. NPJ Park. Dis. 2, 16018 (2016).

101. Olde Dubbelink KTE, Hillebrand A, Stoffers D et al. Disrupted brain network topology in Parkinson's disease: a longitudinal magnetoencephalography study. Brain 137(Pt 1), 197-207 (2014).

102. Hattori T, Orimo S, Aoki S et al. Cognitive status correlates with white matter alteration in Parkinson's disease. Hum. Brain Mapp. 33(3), 727-739 (2012).

103. Pagonabarraga J, Corcuera-Solano I, Vives-Gilabert Y et al. Pattern of regional cortical thinning associated with cognitive deterioration in Parkinson's disease. PLoS ONE 8(1), e54980 (2013).

104. Baggio H-C, Segura B, Sala-Llonch R et al. Cognitive impairment and resting-state network connectivity in Parkinson's disease. Hum. Brain Mapp. 36(1), 199-212 (2015).

105. Zheng Z, Shemmassian S, Wijekoon C, Kim W, Bookheimer SY, Pouratian N. DTI correlates of distinct cognitive impairments in Parkinson's disease. Hum. Brain Mapp. 35(4), 1325-1333 (2014).

106. Wiltshire K, Concha L, Gee M, Bouchard T, Beaulieu C, Camicioli R. Corpus callosum and cingulum tractography in Parkinson's disease. Can. J. Neurol. Sci. 37(5), 595-600 (2010).

107. Kamagata K, Motoi Y, Abe O et al. White matter alteration of the cingulum in Parkinson disease with and without dementia: evaluation by diffusion tensor tract-specific analysis. AJNR Am. J. Neuroradiol. 33(5), 890-895 (2012).

108. Duncan GW, Firbank MJ, Yarnall AJ et al. Gray and white matter imaging: a biomarker for cognitive impairment in early Parkinson's disease? Mov. Disord. 31(1), 103-110 (2016).

109. Nagano-Saito A, Habak C, Mejia-Constain B et al. Effect of mild cognitive impairment on the patterns of neural activity in early Parkinson's disease. Neurobiol. Aging 35(1), 223-231 (2014).

110. Ekman U, Eriksson J, Forsgren L et al. Longitudinal changes in task-evoked brain responses in Parkinson's disease patients with and without mild cognitive impairment. Front. Neurosci. 8, 207 (2014).

111. Redgrave P, Rodriguez M, Smith Y et al. Goal-directed and habitual control in the basal ganglia: implications for Parkinson's disease. Nat. Rev. Neurosci. 11(11), 760-772 (2010).

112. Lewis SJG, Dove A, Robbins TW, Barker RA, Owen AM. Cognitive impairments in early Parkinson's disease are accompanied by reductions in activity in frontostriatal neural circuitry. J. Neurosci. 23(15), 6351-6356 (2003).

113. Amboni M, Tessitore A, Esposito F et al. Resting-state functional connectivity associated with mild cognitive impairment in Parkinson's disease. J. Neurol. 262(2), 425-434 (2015).

114. Christopher L, Marras C, Duff-Canning S et al. Combined insular and striatal dopamine dysfunction are associated with executive deficits in Parkinson's disease with mild cognitive impairment. Brain 137(Pt 2), 565-575 (2014).

115. Sridharan D, Levitin DJ, Menon V. A critical role for the right fronto-insular cortex in switching between central-executive and default-mode networks. Proc. Natl Acad. Sci. USA 105(34), 12569-12574 (2008).

116. Anticevic A, Cole MW, Murray JD, Corlett PR, Wang X-J, Krystal JH. The role of default network deactivation in cognition and disease. Trends Cogn. Sci. 16(12), 584-592 (2012).

117. Klein JC, Eggers C, Kalbe E et al. Neurotransmitter changes in dementia with Lewy bodies and Parkinson disease dementia in vivo. Neurology 74(11), 885-892 (2010).

118. Niethammer M, Tang CC, Ma Y et al. Parkinson's disease cognitive network correlates with caudate dopamine. Neuroimage 78, 204-209 (2013).

119. Monchi O, Petrides M, Doyon J, Postuma RB, Worsley K, Dagher A. Neural bases of set-shifting deficits in Parkinson's disease. J. Neurosci. 24(3), 702-710 (2004).

120. Marie RM, Barre L, Dupuy B, Viader F, Defer G, Baron JC. Relationships between striatal dopamine denervation and frontal executive tests in Parkinson's disease. Neurosci. Lett. 260(2), 77-80 (1999).

121. Sawamoto N, Piccini P, Hotton G, Pavese N, Thielemans K, Brooks DJ. Cognitive deficits and striato-frontal dopamine release in Parkinson's disease. Brain 131(Pt 5), 1294-1302 (2008).

122. Ko JH, Antonelli F, Monchi O et al. Prefrontal dopaminergic receptor abnormalities and executive functions in Parkinson's disease. Hum. Brain Mapp. 34(7), 1591-1604 (2013).

123. Jokinen P, Karrasch M, Bruck A, Johansson J, Bergman J, Rinne JO. Cognitive slowing in Parkinson's disease is related to frontostriatal dopaminergic dysfunction. J. Neurol. Sci. 329(1-2), 23-28 (2013).

124. Christopher L, Duff-Canning S, Koshimori Y et al. Salience network and parahippocampal dopamine dysfunction in memory-impaired Parkinson disease. Ann. Neurol. 77(2), 269-280 (2015).

125. Bentin S, Silverberg R, Gordon HW. Asymmetrical cognitive deterioration in demented and Parkinson patients. Cortex 17(4), 533-543 (1981). 
126. Claassen DO, McDonell KE, Donahue M et al. Cortical asymmetry in Parkinson's disease: early susceptibility of the left hemisphere. Brain Behav. 6(12), e00573 (2016).

127. Gotts SJ, Jo HJ, Wallace GL, Saad ZS, Cox RW, Martin A. Two distinct forms of functional lateralization in the human brain. Proc. Natl Acad. Sci. USA 110(36), E3435-44 (2013).

128. Fleck JI, Kuti J, Brown J, Mahon JR, Gayda-Chelder C. Frontal-posterior coherence and cognitive function in older adults. Int. J. Psychophysiol. 110, 217-230 (2016).

129. Vallesi A. Organisation of executive functions: hemispheric asymmetries. J. Cogn. Psychol.24(4), 367-386 (2012).

130. Nunez PL, Srinivasan R. Electric Fields of the Brain: The Neurophysics of EEG (2nd Edition), Oxford University Press, NY, USA (2005).

131. Srinivasan R, Winter WR, Ding J, Nunez PL. EEG and MEG coherence: measures of functional connectivity at distinct spatial scales of neocortical dynamics. J. Neurosci. Methods 166(1), 41-52 (2007).

132. David O, Garnero L, Cosmelli D, Varela FJ. Estimation of neural dynamics from MEG/EEG cortical current density maps: application to the reconstruction of large-scale cortical synchrony. IEEE Trans. Biomed. Eng. 49(9), 975-987 (2002).

133. Darvas F, Pantazis D, Kucukaltun-Yildirim E, Leahy RM. Mapping human brain function with MEG and EEG: methods and validation. Neuroimage 23(Suppl 1), S289-99 (2004).

134. Schoffelen JM, Gross J. Source connectivity analysis with MEG and EEG. Hum. Brain Mapp.30 (2009).

135. Williams-Gray CH, Mason SL, Evans JR et al. The CamPaIGN study of Parkinson's disease: 10-year outlook in an incident population-based cohort. J. Neurol. Neurosurg. Psychiatry 84(11), 1258-1264 (2013). 